\title{
Achieving Low Latency Two-Way Communication by Downlink and Uplink Decoupled Access
}

\author{
Dong Min Kim, Nuno K. Pratas and Petar Popovski \\ Department of Electronic Systems, Aalborg University, Denmark \\ Email: \{dmk;nup;petarp\}@es.aau.dk
}

\begin{abstract}
】
Abstract-In many scenarios, low latency wireless communication assumes two-way connection, such that the node that receives information can swiftly send acknowledgment or other response. In this paper, we address the problem of low latency two-way communication and address it through proposal of a base station (BS) cooperation scheme. The scheme is based on downlink (DL) and uplink (UL) decoupled access (DUDA). To the best of our knowledge, this is the first time that the idea of decoupled access is used to reduce latency. We derive the analytical expression for the average latency and verify that the latency expression is valid with outage probability based on stochastic geometry analysis. Both analytical and simulation results show that, with DUDA, the latency can be reduced by approximately 30-60\% compared to the traditional coupled access.
\end{abstract}

Index Terms-Low-latency, reliable communications, two-way traffic, stochastic geometry.

\section{INTRODUCTION}

One of the most promising use cases of the emerging $5 \mathrm{G}$ wireless systems is the one of reliable low latency communications [1], [2], such as vehicle-to-vehicle (V2V) and vehicleto-anything (V2X) connections [3], ultra-reliable connections in industrial environments [4]. A reliable communication link is established through a two-way communication protocol, where the receiver always acknowledges the reception of the transmitter's packet. This brings forward the need to have two-way communication links where each device can quickly switch between transmission and reception. The obvious way to achieve low latency is to use full duplex transceivers through the use of Frequency Division Duplex (FDD) systems. Contrary to this, many ongoing efforts are currently favoring Time Division Duplex (TDD) operation in order to reduce transceiver cost, increase spectrum usage efficiency, take advantage of channel reciprocity and to be capable to adapt to time-varying uplink/downlink traffic asymmetries [5]. However, the frame-based structure of TDD is not aligned with the requirement for latency reduction due to the long time it takes to switch between uplink and downlink. Based on these observations, we conclude that there is a need to design low

This work has been in part supported by the European Research Council (ERC Consolidator Grant Nr. 648382 WILLOW) within the Horizon 2020 Program. Part of this work has been performed in the framework of the Horizon 2020 project ONE5G (ICT - 760809) receiving funds from the European Union. The authors would like to acknowledge the contributions of their colleagues in the project, although the views expressed in this contribution are those of the authors and do not necessarily represent the project.
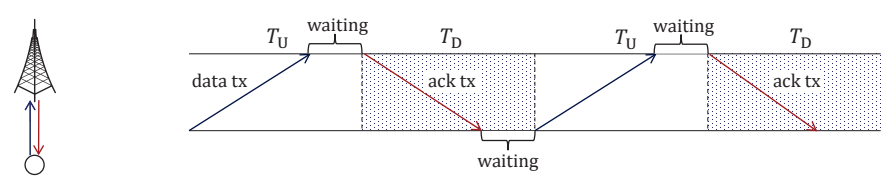

(a)

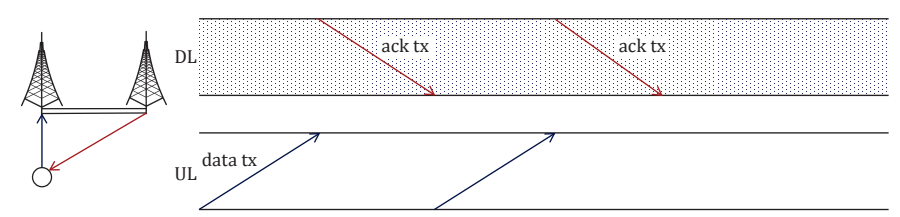

(b)

Fig. 1: Two way traffic initiated in the uplink: (a) $T_{\mathrm{U}}$ denotes uplink frame duration and $T_{\mathrm{D}}$ donwlink frame duration; (b) Proposed scheme with two half-duplex base-stations, where one takes the role of uplink and the other of downlink.

latency two-way communication solutions that can work with terminals (devices) that operate in TDD.

In TDD cellular systems, the minimization of the latency experienced by a two-way communication transaction is constrained by the time period (frame duration) that the halfduplex base station stays in the downlink and uplink directions as shown in Fig. 1a The direct approach is to decrease the time period before shifting from uplink (downlink) to downlink (uplink) direction, in order to facilitate fast interaction between the communicating devices/nodes [6], [7]. This fast switching between uplink and downlink comes at the cost of more complex transceivers both at the device and base station, due to the need to perform faster and more frequent channel estimation, as well as additional signaling overhead, due to the resource assignment.

This paper proposes a radically different method for enabling low-latency two-way communication in frame-based TDD cellular systems. We propose to use two half-duplex base stations instead of one, where the first base station takes the downlink direction and the second the uplink direction (or vice versa). As a motivation example on how this approach can satisfy the individual low-latency requirements, consider the example in Fig. 1a with two devices where each should receive a reply from their original transmission within 2 timeslots. The TDD structure of the baseline scheme in Fig. 1a is not capable of avoiding additional waiting times; while, 
as shown in Fig. 1b, the proposed scheme can support the desired latency, as it allows each device to switch between uplink and downlink directions without requiring the network infrastructure to do so. This of course assumes that the processing time within the device to switch between UL and DL directions is lower than the residual time remaining in the traditional cellular TDD to switch between UL and DL (and vice-versa).

The proposed scheme can be applied both in a traditional and in a cloud radio access network (C-RAN) architecture. In the traditional architecture, the coordination between the half-duplex base stations can be accomplished through the $\mathrm{X} 2$ interface; while in a C-RAN architecture this coordination is implicit at the C-RAN's base-band unit (BBU). The interference resulting from the downlink to the uplink halfduplex base stations can be mitigated by: (i) Taking advantage of spatial pre-computing, beamforming and full-dimension MIMO to steer the downlink interfering beam from the uplink base station; and/or (ii) take advantage of the X2 interface to exchange the necessary information to cancel any residual wireless interference, with the goal of improving the reliability of the uplink reception in a low latency traffic setting.

The proposed scheme builds upon already on-going efforts in 3GPP such as device multi-connectivity, decoupled uplink and downlink access [8], cooperation between base stations and dynamic TDD. Furthermore, it enables the network to continue its evolution towards a device centric architecture and enable the joint design and scheduling of low latency two-way communications [9].

To the best of our knowledge, this is the first study to apply decoupled access in order to decrease latency. We propose a system design, which enables low latency two-way interactive communications in a TDD regime, without incurring the latency penalties associated with the TDD UL and DL cycling periods. This is of relevance since TDD operation allows to reduce transceiver cost, increase spectrum usage efficiency and adapt to time-varying Uplink/Downlink (UL/DL) traffic asymmetries [5]. We quantify the proposed scheme and we show that the proposed scheme is always able to achieve lower two-way communication latency than the baseline scheme.

The paper is organized as follows. We describe the system model and the proposed scheme in Section II and Section III, respectively. The latency and reliability analysis of proposed scheme is given in Section IV, and its numerical results are presented in Section V. The paper is concluded in Section VI.

\section{SySTEM MOdeL}

In this section, we explain the system model to evaluate the latency performance of two-way traffic in the cellular networks. some basic assumptions are provided in the following subsections.

\section{A. Network and Channel Model}

In a cellular network, the base stations (BSs) are randomly distributed with density $\lambda_{\mathrm{b}}$, resulting in a homogeneous Poisson point process (PPP) [10]. A pair of BSs are interconnected

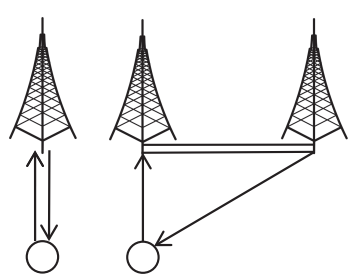

(a)

(b)

Fig. 2: (a) Baseline scheme; (b) Proposed scheme.

via a wired connection (double solid line in Fig. 2b). BSs can serve cross directional traffic, one BS will operate in DL (DLBS) and the other in UL (UL-BS), or vice-versa. The UL-BS can use side information sent from the DL-BS through the wired backhaul. We assume that the closer BS will operate in UL (UL-BS) and the other in DL (DL-BS).

The user equipments (UEs) associate with their nearest BSs first. A single spectrum with unit bandwidth and Rayleigh fading channel with unit mean power is assumed. All transmitted signals experience path loss as follows: $\ell(r)=r^{-\alpha}$, with path loss exponent $\alpha$. The default transmit power of uplink UEs is $P_{\mathrm{m}}$. BSs transmit with constant power $P_{\mathrm{b}}$.

\section{B. Transmission Success and Retransmission Model}

A signal-to-interference-plus-noise ratio (SINR) requirement for uplink and downlink is denoted by $\beta_{\mathrm{u}}$ and $\beta_{\mathrm{d}}$, respectively. The transmission is successful if the received SINR is greater than or equal to the target threshold value:

$$
\begin{aligned}
& \rho_{\mathrm{d}} \triangleq \operatorname{Pr}\left[\operatorname{SINR}_{\mathrm{d}}>\beta_{\mathrm{d}}\right], \\
& \rho_{\mathrm{u}} \triangleq \operatorname{Pr}\left[\operatorname{SINR}_{\mathrm{u}}>\beta_{\mathrm{u}}\right] .
\end{aligned}
$$

Even if the transmission is successful, if the acknowledgement is not received, a retransmission is attempted. Retransmission stops when the maximum number of retransmissions is reached or an acknowledgement arrives. With $n$ transmission opportunities, the transmission success is described as follows:

$$
p_{\mathrm{s}}^{(n)} \triangleq \sum_{i=0}^{n-1}\left(1-\rho_{\mathrm{d}} \rho_{\mathrm{u}}\right)^{i} \rho_{\mathrm{d}} \rho_{\mathrm{u}}=1-\left(1-\rho_{\mathrm{d}} \rho_{\mathrm{u}}\right)^{n} .
$$

\section{Traffic and Association Model}

In this paper, we consider a situation where data is transmitted on the UL and its acknowledgment (ACK) is transmitted on the DL. If the UL transmission fails, the BS does not transmit an ACK, so the UE waits for the ACK for a certain period of time and retransmits the data. The same operation is performed when the $\mathrm{BS}$ transmits an $\mathrm{ACK}$, but this is not decoded successfully at the UE.

In general the transmission power output of the UE is weaker than that of the BS. So, it is efficient to allocate a better channel to the UL transmission. Let us set up that the UE receives the UL transmission from the far BS and transmits the UL data to the nearer BS among two cooperating BSs. 


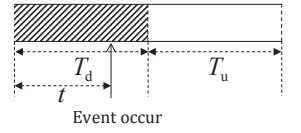

Fig. 3: Illustration of time slot composition.

\section{Proposed Scheme}

The basic principle of our proposal is the introduction of two Half-Duplex Base Stations (HDBS), the first operating in the downlink and the second on the uplink (or vice-versa), as depicted in Fig. 2b. A half-duplex mobile device that connects to the infrastructure, associates simultaneously with both HDBS, such that at one time it receives from one of the HDBSs and at another time it transmits to the other HDBS.

This scheme will permit to reduce the latency of two-way communication transactions beyond what is possible using a traditional TDD system. It should be noted that this is in line with the recent trends of decoupled uplink/downlink access [8], device multi-connectivity, cooperation between base stations and dynamic TDD; however, the instance and the benefit presented in this paper have not been observed so far.

In TDD systems, the proposed scheme, where the HalfDuplex Base Stations (HDBSs) are connected via an X2 interface (Fig. 2b), can achieve lower latencies than the single TDD baseline (Fig. 2a). The baseline scheme is composed by a half-duplex device and a HDBS as shown in Fig. 2a The simplest realization of the proposed scheme is depicted in Fig. 2b. Each transceiver on the picture, both at the UE and at the infrastructure, is half-duplex and operates in a TDD mode.

\section{LATENCY AND RELIABILITY ANALYSIS}

In the following we show that the latency analysis of the baseline and proposed scheme.

\section{A. Latency Model}

1) Protocol delay: For TDD system, the protocol delay is the waiting time until the transmission slot arrives. The time is discretized into downlink and uplink time slot with durations $T_{\mathrm{d}}$ and $T_{\mathrm{u}}$, respectively.

We assume that the packet is generated randomly and uniformly over the time at the UE. If a packet with size1 $S_{\mathrm{u}}\left(0<S_{\mathrm{u}} \leq T_{\mathrm{u}}\right)$ is generated after $t\left(0 \leq t \leq T_{\mathrm{d}}+T_{\mathrm{u}}\right)$ time from the start of the DL slot, the protocol latency $L_{\mathrm{p}}$ can be described as:

$$
\begin{aligned}
L_{\mathrm{p}} & =\left(T_{\mathrm{d}}-t\right) \cdot \operatorname{Pr}\left(t \leq T_{\mathrm{d}}\right)+0 \cdot \operatorname{Pr}\left(T_{\mathrm{d}}<t \leq T_{\mathrm{d}}+T_{\mathrm{u}}-S_{\mathrm{u}}\right) \\
& +\left(T_{\mathrm{d}}+T_{\mathrm{u}}-t+T_{\mathrm{d}}\right) \cdot \operatorname{Pr}\left(T_{\mathrm{d}}+T_{\mathrm{u}}-S_{\mathrm{u}}<t \leq T_{\mathrm{d}}+T_{\mathrm{u}}\right) \\
& =\left(T_{\mathrm{d}}-t\right) \frac{T_{\mathrm{d}}}{T_{\mathrm{d}}+T_{\mathrm{u}}}+\left(2 T_{\mathrm{d}}+T_{\mathrm{u}}-t\right) \frac{S_{\mathrm{u}}}{T_{\mathrm{d}}+T_{\mathrm{u}}}
\end{aligned}
$$

\footnotetext{
${ }^{1}$ The size of the packet is measured by the amount time it occupies.
}

The number of transmissions until success can be modeled by geometric distribution. Then the retransmission delay $L_{\mathrm{r}}$ can be described as:

$$
L_{\mathrm{r}}=\left(T_{\mathrm{d}}+T_{\mathrm{u}}\right) \cdot\left(\frac{1}{\rho_{\mathrm{u}} \rho_{\mathrm{d}}}-1\right) .
$$

Even without protocol delay and retransmission delay, there is a fundamental delay $L_{\mathrm{f}}$ caused from the transmission delay and reception delay. If a size of the acknowledgment in downlink is $S_{\mathrm{d}}\left(0<S_{\mathrm{d}} \leq T_{\mathrm{d}}\right)$, the fundamental delay can be described as:

$$
L_{\mathrm{f}}=T_{\mathrm{u}}+S_{\mathrm{d}} .
$$

Then, the total expected latency of TDD scheme (or downlink and uplink coupled access (DUCA)) $L_{\mathrm{DUCA}}$ is:

$$
\begin{aligned}
L_{\mathrm{DUCA}}= & \mathbb{E}\left[L_{\mathrm{p}}+L_{\mathrm{r}}+L_{\mathrm{f}}\right] \\
= & \frac{T_{\mathrm{d}}^{2}+\left(2 T_{\mathrm{d}}+T_{\mathrm{u}}\right) S_{\mathrm{u}}}{T_{\mathrm{d}}+T_{\mathrm{u}}}-\frac{T_{\mathrm{d}}+S_{\mathrm{u}}}{2} \\
& +\left(T_{\mathrm{d}}+T_{\mathrm{u}}\right)\left(\frac{1}{\rho_{\mathrm{u}} \rho_{\mathrm{d}}}-1\right)+T_{\mathrm{u}}+S_{\mathrm{d}} .
\end{aligned}
$$

For the proposed scheme, because the user can transmit its packet whenever, there is no protocol delay caused by fixed time division duplex. Then, only retransmission delay and fundamental delay remain. But there is a waiting time $W$ for ACK. It is fair to set this time period as same as $T_{\mathrm{d}}$ :

$$
\begin{aligned}
L_{\text {DUDA }} & =\left(S_{\mathrm{u}}+W\right)\left(\frac{1}{\rho_{\mathrm{u}} \rho_{\mathrm{d}}}-1\right)+S_{\mathrm{u}}+S_{\mathrm{d}} \\
& =\left(S_{\mathrm{u}}+T_{\mathrm{d}}\right)\left(\frac{1}{\rho_{\mathrm{u}} \rho_{\mathrm{d}}}-1\right)+S_{\mathrm{u}}+S_{\mathrm{d}} .
\end{aligned}
$$

Let us compute $L_{\mathrm{DUCA}}-L_{\mathrm{DUDA}}$. With assuming $T_{\mathrm{d}}=T_{\mathrm{u}}$ to be fair.

$$
L_{\mathrm{DUCA}}-L_{\mathrm{DUDA}}=\frac{T_{\mathrm{u}}-S_{\mathrm{u}}}{\rho_{\mathrm{u}} \rho_{\mathrm{d}}}+S_{\mathrm{u}}>0 .
$$

So, the proposed scheme is always able to achieve lower twoway communication latency than the baseline scheme.

\section{B. Reliability analysis}

In this subsection, we investigate the reliability performance by deriving analytical expressions for the transmission success probabilities in UL and DL using stochastic geometry. For the analytical tractability, we assume that all BSs in the network can schedule one UE either UL (UL-UE) or DL (DL-UE) in each Voronoi cell for the TDD case. For the proposed scheme, a pair of cooperating BSs will serve only one UE at a time. We further assume that the spatial distribution of UEs follows another independent PPP with the density $\lambda_{\mathrm{b}}$.

1) UL Success Probability in DUDA Network: The success probability of the transmission of a typical UL user $\mathcal{U}$ at a typical BS $B(\mathcal{U})$ in DUDA $p_{\mathrm{U}}^{\mathrm{DUDA}}$ can be expressed as follows:

$$
\begin{aligned}
p_{\mathrm{U}}^{\text {DUDA }} & =\mathbb{E}\left[\operatorname{Pr}\left[\frac{g_{\mathcal{U}, B(\mathcal{U})} r^{-\alpha} P_{\mathrm{m}}}{I_{B(\mathcal{U})}^{\psi}+I_{B(\mathcal{U})}^{\varphi}} \geq \beta_{\mathrm{u}}\right]\right] \\
& =\mathbb{E}\left[\operatorname{Pr}\left[g_{\mathcal{U}, B(\mathcal{U})} \geq \frac{\beta_{\mathrm{u}} r^{\alpha}}{P_{\mathrm{m}}}\left(I_{B(\mathcal{U})}^{\psi}+I_{B(\mathcal{U})}^{\varphi}\right)\right]\right],
\end{aligned}
$$


where $g_{i, j}$ denotes the gain at node $j$ of the channel from node $i$, and $I_{i}^{\psi}$ and $I_{i}^{\varphi}$ denote the aggregate interference at node $i$ from DL-BSs and UL-UEs, respectively. The UL transmission distance between the typical user and the typical BS is denoted by $r$, and $r$ is assumed as random variable with pdf as $f(r)=$ $2 \pi \lambda_{\mathrm{b}} r \exp \left(-\pi \lambda_{\mathrm{b}} r^{2}\right)$ [11]. Due to the Rayleigh faded channel, $g_{\mathcal{U}, B(\mathcal{U})}$ is an exponential random variable with unit mean, then (10) can be expressed as:

$$
\begin{aligned}
p_{\mathrm{U}}^{\mathrm{DUDA}} & =\mathbb{E}_{r}\left[\mathbb{E}_{I_{B(\mathcal{U})}^{\psi}, I_{B(\mathcal{U})}^{\varphi}}\left[\exp \left(-s\left(I_{B(\mathcal{U})}^{\psi}+I_{B(\mathcal{U})}^{\varphi}\right)\right)\right]\right] \\
& =\mathbb{E}_{r}\left[\mathbb { E } _ { I _ { B ( \mathcal { U } ) } ^ { \psi } } [ e ^ { - s I _ { B ( \mathcal { U } ) } ^ { \psi } ] } ] \mathbb { E } _ { I _ { B ( \mathcal { U } ) } ^ { \varphi } } \left[e^{\left.\left.-s I_{B(\mathcal{U})}^{\varphi}\right]\right]}\right.\right. \\
& =\int_{0}^{\infty} \mathcal{L}_{\mathrm{U}}^{\psi}(s) \mathcal{L}_{\mathrm{U}}^{\varphi}(s) 2 \pi \lambda_{\mathrm{b}} r \exp \left(-\pi \lambda_{\mathrm{b}} r^{2}\right) d r,
\end{aligned}
$$

where $s=\frac{\beta_{\mathrm{u}} r^{\alpha}}{P_{\mathrm{m}}}$, and $\mathcal{L}_{\mathrm{U}}^{\psi}(s)$ and $\mathcal{L}_{\mathrm{U}}^{\varphi}(s)$ are the Laplace functionals of the interference from DL-BSs at the typical BS and the interference from UL-UEs at the typical BS, respectively. The interference from DL-BS is coming from outside of the pair. Assuming independence of the channels from different interfering DL-BSs and independence of the distances from different interfering DL-BSs, and using moment generating function of exponential distribution, $\mathcal{L}_{\mathrm{U}}^{\psi}(s)$ can be expressed as:

$$
\begin{aligned}
& \mathcal{L}_{\mathrm{U}}^{\psi}(s)=\mathbb{E}_{I_{B(\mathcal{U})}^{\psi}}\left[\exp \left(-\frac{\beta_{\mathrm{u}} r^{\alpha}}{P_{\mathrm{m}}} I_{B(\mathcal{U})}^{\psi}\right)\right] \\
& =\mathbb{E}_{g_{i, B(\mathcal{U})}, r_{i, B(\mathcal{U})}}\left[\exp \left(-\frac{\beta_{\mathrm{u}} r^{\alpha}}{P_{\mathrm{m}}} \sum_{i \in \Phi^{\psi}} g_{i, B(\mathcal{U})} r_{i, B(\mathcal{U})}^{-\alpha} P_{\mathrm{b}}\right)\right] \\
& =\mathbb{E}_{r_{i, B(\mathcal{U})}}\left[\prod_{i \in \Phi^{\psi}} \mathbb{E}_{g_{i, B(\mathcal{U})}}\left[\exp \left(-\frac{P_{\mathrm{b}}}{P_{\mathrm{m}}} g_{i, B(\mathcal{U})} \beta_{\mathrm{u}} r^{\alpha} r_{i, B(\mathcal{U})}^{-\alpha}\right)\right]\right] \\
& =\mathbb{E}_{r_{i, B(\mathcal{U})}}\left[\prod_{i \in \Phi^{\psi}} \frac{1}{1+\left(P_{\mathrm{b}} / P_{\mathrm{m}}\right) \beta_{\mathrm{u}} r^{\alpha} r_{i, B(\mathcal{U})}^{-\alpha}}\right]
\end{aligned}
$$

where $r_{i, B(\mathcal{U})}$ denotes the distance from $i$ th BS in the interfering BS set $\Phi^{\psi}$ to the typical BS $B(\mathcal{U})$. To model the density of interfering nodes, we define $\delta$ as the ratio of DL traffic of the entire network. The traffic asymmetry can be modeled by adjusting $\delta$. The range of $\delta$ is $(0,1)$. We assume that the value of $\delta$ is not changed over the observation period even though the real transmitting nodes are varying. The average node density of DL-BSs is $\delta \lambda_{\mathrm{b}}$. Because a pair of cooperating BSs will serve one user, the higher achievable node density of the DLBS is $0.5 \lambda_{\mathrm{b}}$. We assume that the set $\Phi^{\psi}$ follows a PPP with density $\lambda_{\mathrm{b}}^{\psi}$, where $\lambda_{\mathrm{b}}^{\psi}=0.5 \delta \lambda_{\mathrm{b}}$. Assuming the paired BS is the nearest DL-BS of the UL-BS, the distance to the nearest interfering $\mathrm{BS}$ is the distance to the second nearest DL-BS. The second nearest distance distribution is given as [12]:

$$
f(d)=2(\pi \lambda)^{2} d^{3} \exp \left(-\pi \lambda d^{2}\right)
$$

Using (13), $\lambda_{\mathrm{b}}^{\psi}$, and probability generating functional (PGFL) of PPP [10], 112 can be expressed as:

$$
\begin{gathered}
\mathcal{L}_{\mathrm{U}}^{\psi}(s)=\int_{0}^{\infty} \exp \left(-2 \pi \lambda_{\mathrm{b}}^{\psi} \int_{t}^{\infty} \frac{\left(P_{\mathrm{b}} / P_{\mathrm{m}}\right) \beta_{\mathrm{u}} r^{\alpha} x^{-\alpha}}{1+\left(P_{\mathrm{b}} / P_{\mathrm{m}}\right) \beta_{\mathrm{u}} r^{\alpha} x^{-\alpha}} x d x\right) . \\
2\left(\pi \lambda_{\mathrm{b}}\right)^{2} t^{3} \exp \left(-\pi \lambda_{\mathrm{b}} t^{2}\right) d t
\end{gathered}
$$

where $t$ is the distance to the nearest interfering DL-BS (second nearest BS). For brevity, $r_{i, B(\mathcal{U})}$ is changed as $x$.

In a similar way, we can obtain $\mathcal{L}_{\mathrm{U}}^{\varphi}(s)$. The interfering MS set will be denoted $\Phi^{\varphi}$ and it is assumed as PPP with density $\lambda_{\mathrm{b}}^{\varphi}$. The average node density of UL-MSs is $0.5(1-\delta) \lambda_{\mathrm{b}}$. So, $\lambda_{\mathrm{b}}^{\varphi}$ is equal to $0.5(1-\delta) \lambda_{\mathrm{b}}$. Then $\mathcal{L}_{\mathrm{U}}^{\varphi}(s)$ can be expressed as:

$$
\mathcal{L}_{\mathrm{U}}^{\varphi}(s)=\exp \left(-2 \pi \lambda_{\mathrm{b}}^{\varphi} \int_{r}^{\infty} \frac{\beta_{\mathrm{u}} r^{\alpha} y^{-\alpha}}{1+\beta_{\mathrm{u}} r^{\alpha} y^{-\alpha}} y d y\right),
$$

where $y$ denotes the distance from interfering UL-MSs to the typical BS. It is assumed that the interfering UL-MSs are located at a distance larger than $r$. Using (10), (14), and (15), we can evaluate the UL transmission success probability for DUDA.

\section{DL Success Probability in DUDA Network}

Following the same lines as $p_{\mathrm{U}}^{\mathrm{DUDA}}$, we can obtain the analytical expression for the DL transmission success probability as follows:

$$
p_{\mathrm{D}}^{\mathrm{DUDA}} \approx \int_{0}^{\infty} \mathcal{L}_{\mathrm{D}}^{\psi}(s) \mathcal{L}_{\mathrm{D}}^{\varphi}(s) 2 \pi \lambda_{\mathrm{b}}{ }^{2} r^{3} \exp \left(-\pi \lambda_{\mathrm{b}} r^{2}\right) d r
$$

where $s=\frac{\beta_{\mathrm{d}} r^{\alpha}}{P_{\mathrm{b}}}$ and the Laplace functionals of the interference from BSs $\mathcal{L}_{\mathrm{D}}^{\psi}(s)$ and MSs $\mathcal{L}_{\mathrm{D}}^{\varphi}(s)$ are

$$
\begin{gathered}
\mathcal{L}_{\mathrm{D}}^{\psi}(s)=\exp \left(-2 \pi \lambda_{\mathrm{b}}^{\psi} \int_{r}^{\infty}\left(\frac{\beta_{\mathrm{d}} r^{\alpha} x^{-\alpha}}{1+\beta_{\mathrm{d}} r^{\alpha} x^{-\alpha}}\right) x d x\right), \\
\mathcal{L}_{\mathrm{D}}^{\varphi}(s)=\exp \left(-2 \pi \lambda_{\mathrm{b}}^{\varphi} \int_{0}^{\infty} \frac{\left(P_{\mathrm{m}} / P_{\mathrm{b}}\right) \beta_{\mathrm{d}} r^{\alpha} y^{-\alpha}}{1+\left(P_{\mathrm{m}} / P_{\mathrm{b}}\right) \beta_{\mathrm{d}} r^{\alpha} y^{-\alpha}} y d y\right) .
\end{gathered}
$$

The distance from the interfering DL-BSs to the typical DL-MS $x$ cannot be closer than $r$ as shown in [11]. We further apply this distance restriction to the distance from the interfering UL-MSs to the typical DL-MS $y$ to approximate.

\section{Numerical Results}

We quantify the latency performance of DUDA, where the analytical results of (10) and (16) are compared with numerical simulations. All the simulation results are obtained by performing Monte Carlo simulation with 10000 iterations. The common parameters used are shown in Table 【.

The analysis from (9) implied the proposed method has superior performance in terms of latency, measured in time slots. This is further verified through a simulation, conducted in a two-dimensional spatial region, following the principles 


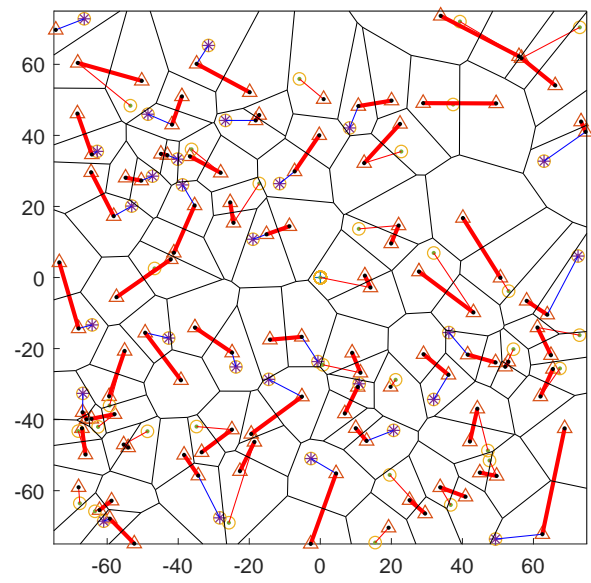

Fig. 4: A snapshot of proposed scheme in two dimensional network.

for wireless network evaluation through stochastic geometry models. The simulation is built as follows:

1) Deploy HDBSs according to Poisson point process (PPP) with intensity $\lambda_{\mathrm{b}}$. The typical node (DL: UE, UL: BS) is located at the origin.

2) Construct Voronoi cell.

3) Starting from one HDBS randomly, search nearest HDBS among HDBSs who are sharing the same edges.

4) Make a pair if the HDBS is unpaired.

5) For the interfering BS pairs, randomly generate transmission direction according to $\delta$ and deploy active UE in one of cells of cooperating BSs.

6) Repeat the procedure 3)-5) for the other HDBS randomly.

7) Calculate SINR and until two-way transmission succeeds.

8) Record latency and return to step 1) and repeat 10000 times.

Only one of the cooperating BSs has an active UE to make two BSs serving one UE. A snapshot of the deployment is as shown in Fig. 4.

In Fig. 5, we validate the our latency analysis with the normalized data length. Because the data slot length is one,

TABLE I: Simulation parameters.

\begin{tabular}{clc}
\hline Parameter & Description & Simulation Setting \\
\hline$S$ & Size of observation window & $150 \mathrm{~m}$ \\
$\lambda_{\mathrm{b}}$ & BS density & $0.005 \mathrm{BS} / \mathrm{m}^{2}$ \\
$\delta$ & Traffic asymmetry ratio & 0.5 \\
$\sigma^{2}$ & Noise power at MS and BS & $-174 \mathrm{dBm}$ \\
$\alpha$ & Path loss exponent & 4 \\
$\beta_{\mathrm{d}}$ & DL SINR threshold (ACK) & $-5 \mathrm{~dB}$ \\
$\beta_{\mathrm{u}}$ & UL SINR threshold (Data) & $0 \mathrm{~dB}$ \\
$P_{\mathrm{b}}$ & BS transmission power & $40 \mathrm{dBm}$ \\
$P_{\mathrm{m}}$ & MS transmission power & $20 \mathrm{dBm}$ \\
$W$ & System bandwidth & $1 \mathrm{~Hz}$ \\
$N$ & Simulation iterations & 10000
\end{tabular}

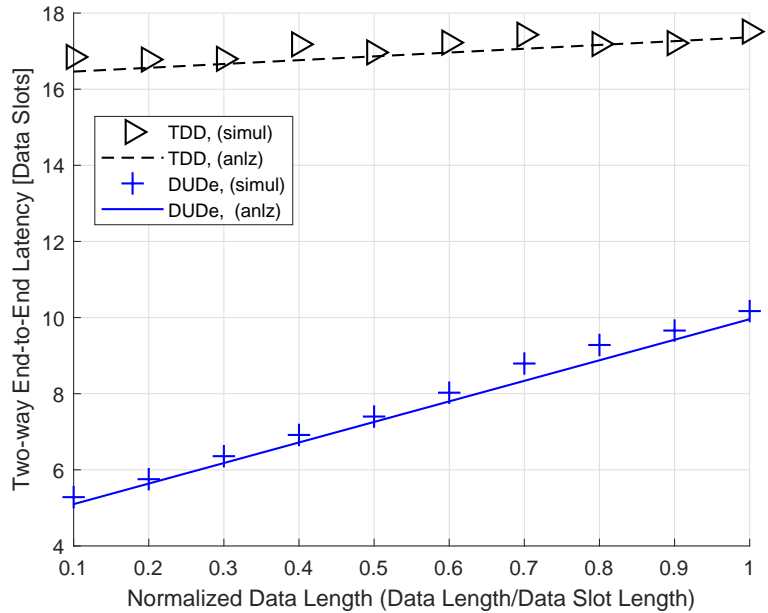

Fig. 5: Two-way latency as a function of normalized data length.

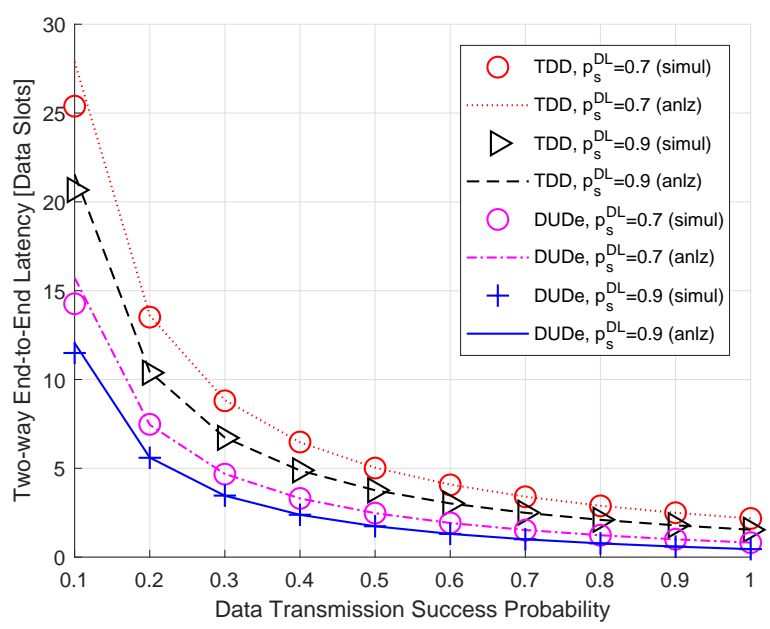

Fig. 6: Two-way latency as a function of data transmission success probability.

the normalized data length has the value in $(0,1)$ range. As the normalized data length becomes longer, the transmission time increases. Therefore, it is natural that transmission delay increases in both DUCA and DUDA in proportion to the normalized data length. However, in the case of DUDA, it shows shorter latency of about 30-60\% compared to DUCA. This is because there is no time slot switching time in twoway transmission. In the case of DUCA the UE always communicates with the nearest BS, which means that UE and BS are the optimal combination. However, the DUDA communicates with the nearest BS in UL, but not in DL. As we can see from Fig. 5, this seems to have little effect.

As we have seen the above, DUDA does not always utilize the topology that produces optimal communication. That is, in some cases the probability of successful transmission may be very low. The impact of the transmission success probability 
is shown on Fig. 6. The two-way latency is expressed as a function of the transmission success probability. The latency is inversely proportional to the success probability of the data transmission, since additional retransmissions are required if the lower is the success transmissions of the data transmission. Both DUCA and DUDA show similar trends. However, in all cases where the simulation was performed, the DUDA exhibits a lower latency than the DUCA. The difference in performance gap between the two is larger when the transmission success probability is low. This matches the expected behaviour as it was shown in the derivation of (9).

\section{CONCLUding REMARKS}

In this paper, the latency performance of the downlink and uplink decoupled access (DUDA) was investigated. We verified that the latency expression is valid with outage probability based on stochastic geometry analysis. Both analytical and simulation results showed that the latency performance is improved with DUDA. The proposed scheme can be applied to improve the current ongoing LTE-TDD and its evolution towards $5 \mathrm{G}$.

\section{REFERENCES}

[1] ITU-R, "IMT vision - framework and overall objectives of the future development for IMT for 2020 and beyond," Recommendation ITU-R M.2083-0, Sep. 2015.

[2] 3GPP, "Study on new radio (NR) access technology physical layer aspects," TR 38.802, Mar. 2017.

[3] M. I. Ashraf, C.-F. Liu, M. Bennis, and W. Saad, "Towards low-latency and ultra-reliable vehicle-to-vehicle communication," in Networks and Communications (EuCNC), 2017 European Conference on. IEEE, 2017, pp. $1-5$.

[4] P. Schulz, M. Matthe, H. Klessig, M. Simsek, G. Fettweis, J. Ansari, S. A. Ashraf, B. Almeroth, J. Voigt, I. Riedel et al., "Latency critical IoT applications in 5G: Perspective on the design of radio interface and network architecture," IEEE Commun. Mag., vol. 55, no. 2, pp. 70-78, Feb. 2017.

[5] Z. Shen, A. Khoryaev, E. Eriksson, and X. Pan, "Dynamic uplinkdownlink configuration and interference management in td-lte," IEEE Commun. Mag., vol. 50, no. 11, pp. 51-59, Nov. 2012.

[6] N. H. Mahmood, M. Lauridsen, G. Berardinelli, D. Catania, and P. Mogensen, "Radio resource management techniques for embb and mmtc services in $5 \mathrm{~g}$ dense small cell scenarios," in IEEE Vehicular Technology Conference (VTC-Fall), 2016, Sep. 2016.

[7] G. Pocovi, K. I. Pedersen, B. Soret, M. Lauridsen, and P. Mogensen, "On the impact of multi-user traffic dynamics on low latency communications," in Wireless Communication Systems (ISWCS), 2016 International Symposium on. IEEE, 2016, pp. 204-208.

[8] F. Boccardi, J. Andrews, H. Elshaer, M. Dohler, S. Parkvall, P. Popovski, and S. Singh, "Why to decouple the uplink and downlink in cellular networks and how to do it," IEEE Commun. Mag., vol. 54, no. 3, pp. 110-117, Mar. 2016.

[9] P. Popovski, O. Simeone, J. J. Nielsen, and C. Stefanovic, "Interference spins: Scheduling of multiple interfering two-way wireless links," IEEE Commun. Lett., vol. 19, no. 3, pp. 387-390, Mar. 2015.

[10] S. N. Chiu, D. Stoyan, W. S. Kendall, and J. Mecke, Stochastic Geometry and its Applications, 3rd ed. Wiley, 2013.

[11] J. G. Andrews, F. Baccelli, and R. K. Ganti, "A tractable approach to coverage and rate in cellular networks," IEEE Trans. Commun., vol. 59, no. 11, pp. 3122-3134, Nov. 2011.

[12] D. Moltchanov, "Distance distributions in random networks," Ad Hoc Networks, vol. 10, no. 6, pp. 1146-1166, Aug. 2012. 